\title{
SUMS OF TWO-DIMENSIONAL SPECTRAL TRIPLES
}

\author{
ERIK CHRISTENSEN and CRISTINA IVAN*
}

(Dedicated to the memory of Gert K. Pedersen)

\begin{abstract}
We study countable sums of two-dimensional modules for the continuous complex functions on a compact metric space and show that it is possible to construct a spectral triple which gives the original metric back. This spectral triple will be finitely summable for any positive parameter. We also construct a sum of two-dimensional modules which reflects some aspects of the topological dimensions of the compact metric space, but this will only give the metric back approximately. At the end we make an explicit computation of the last module for the unit interval in R. The metric is recovered exactly, the Dixmier trace induces a multiple of the Lebesgue integral and the growth of the number of eigenvalues $N(\Lambda)$ bounded by $\Lambda$ behaves, such that $N(\Lambda) / \Lambda$ is bounded, but without limit for $\Lambda \rightarrow \infty$.
\end{abstract}

\section{Introduction}

Gelfand's fundamental theorem on Abelian $C^{*}$-algebras shows that the study of unital commutative $C^{*}$-algebras is the same as the study of compact, Hausdorff, topological spaces. It has been clear for many years that the non commutative $C^{*}$-algebras have many properties in common with their Abelian relatives, for instance the theory of measure and integration was already well developed in the non commutative setting by Murray and von Neumann. The basic difference is of course that for a unital Abelian $C^{*}$-algebras there is a space, the spectrum, which can be investigated via lots of different mathematical theories. Quite a few theories can on the other hand be expressed in terms of certain subalgebras of the algebra of continuous functions on the spectrum. As examples, one may think of differentiability and Lipschitz continuity. Also other structures like K-theory are expressible in terms certain subalgebras of the continuous functions on the spectrum and matrix algebras over such algebras. Especially Alain Connes has tried to express geometrical structures this way in order to be able to extend the classical differential geometry to the non commutative world. In the classical setting a differential geometric structure

* The authors want to thank professor Michel L. Lapidus from University of California at Riverside for much good advice and many interesting discussions on possible applications of the methods from non commutative geometry to the study of the geometry of fractals.

Received January 19, 2006. 
can be viewed as something - extra - added to the compact topological space in question. In the non commutative case one should expect that there is a $C^{*}$ algebra in the background and that the geometrical features such as smooth functions is something extra. In Connes' work [3] this has been synthesized in the concept called a spectral triple, which is defined as

Definition 1.1. Let $\mathscr{A}$ be a unital $C^{*}$-algebra, $H$ a Hilbert space which carries a unital representation $\pi$ of $\mathscr{A}$ and $D$ an unbounded self-adjoint operator on $H$. The set $(\mathscr{A}, H, D)$ is called a spectral triple if

(i) the set $\{a \in \mathscr{A} \mid\|[D, \pi(a)]\|<\infty\}$ is a dense subset of $\mathscr{A}$,

(ii) the operator $\left(I+D^{2}\right)^{-1}$ is compact.

If $\operatorname{tr}\left(\left(I+D^{2}\right)^{-p / 2}\right)<\infty$ for some positive $p$ then the spectral triple is said to be $p$-summable, or just finitely summable.

The set $\{a \in \mathscr{A} \mid\|[D, \pi(a)]\|<\infty\}$ may be thought of as the set of continuous functions which have essentially bounded derivatives, but the concept of a spectral triple is much more than a way to express an analogy. One of the striking results by Connes is the theorem which shows that the geodesic distance on a compact spin Riemannian manifold can be computed via a spectral triple. This setup is described in [4] Chapter VI. The expression which gives the geodesic distance back on a manifold can easily be extended to define a metric on the set of regular Borel probability measures on the manifold. On the other hand such probability measures are exactly what is called states on a $C^{*}$-algebra so we get in this way a metric on the state space of a $C^{*}$-algebra from a spectral triple, [4] VI.1, p. 544:

Definition 1.2. Let $\mathrm{ST}:=(\mathscr{A}, H, D)$ be a spectral triple then the induced metric $d_{\mathrm{ST}}$ on the state space $\mathscr{S}(\mathscr{A})$ is defined by:

$$
\forall \phi, \psi \in \mathscr{S}(\mathscr{A}): d_{\mathrm{ST}}(\phi, \psi):=\sup \{|\phi(a)-\psi(a)| \mid\|[D, \pi(a)]\| \leq 1\} .
$$

It should be remarked that such a metric is non-standard in the way that it may take the value $\infty$ on certain pairs of states, but it is on the other hand fascinating, that the non commutative world keeps track of the metric on a space, which no longer exists in it's classical form.

In the present paper we study especially the metric of compact metric spaces from a non commutative point of view. This kind of investigation has already been performed by Marc Rieffel. In several papers, among which we only cite a few [21], [23], Rieffel studies a general compact metric space by considering a subalgebra of the algebra of continuous functions on the space and a seminorm on this algebra, rather than studying the space and a metric on the space. He also showed how the metric of any compact metric space can be recovered from 
a Dirac operator which is a sort of a differential operator. This construction does not give a spectral triple because the Dirac operator will not have compact resolvent.

Connes has associated the concept called a Dixmier trace to a spectral triple, and shown that in the case of a compact spin Riemannian manifold this induces a functional, which is a multiple of the volume form [4], Formula 2, p. 545. More than 10 years ago Michel L. Lapidus realized that Connes' spectral triples and associated Dixmier traces are important tools in his ongoing search for possible extensions of classical results by Weyl on the asymptotic growth of the eigenvalues of the Laplacian operator on a bounded open subset of $\mathrm{R}^{d}$ with a nice boundary. Lapidus wanted to extend these results to bounded open subsets of $\mathrm{R}^{d}$ with fractal boundaries [12] and even further to fractal subsets of $\mathbf{R}^{d}$ [10], [13], [17], [18]. In these papers the study of the geometry is linked to an investigation of the Laplacian and its spectral distribution. This scope is a little bit different from ours since we are looking at possible Dirac operators, whose squares are supposed to be Laplacians. On the other hand a spectral triple, as constructed in this article, is of a discrete nature, since the associated representation of the algebra of continuous functions takes place inside an algebra of type $\ell^{\infty}$. For a classical differential operator the associated representation of the function algebra is usually as an ultraweakly dense subalgebra of an $L^{\infty}$-algebra. Anyway the results of Weyl can make sense in both settings, since both settings come with Dirac operators with discrete spectra.

In the papers [13], [17], [18] Lapidus, Maier and Pomerance establish a connection between the Riemann Hypothesis and the Weyl-Berry Conjecture for a fractal string. The relations between Riemann's zeta function and fractal geometry is further developed in the book [16] Fractal geometry and number theory, by Lapidus and Frankenhuysen. The possibility to continue this investigation based on Connes spectral triples and the Dixmier trace lies in the fact that besides the connection between the Dirac operator and the Laplacian in the classical case, the zeta function $\zeta(s)$ is a multiple of the trace of the operator $|D|^{-s}$ and the Dixmier trace gives the volume form. The papers [14], [15] contain detailed studies of the ways non commutative geometry can be used to investigate important problems in fractal geometry.

In this paper we are studying abstract compact metric spaces and included here are compact fractal subsets of $\mathbf{R}^{d}$, and we hope that the spectral triple we are proposing in Theorem 3.2 may turn out to be usable in Lapidus' project of studying fractal sets via concepts from non commutative geometry. On the other hand the spectral triples of this articles Theorem 3.1 and Theorem 3.2 will give Laplacians of a quite different nature than the ones studied by Lapidus and his coauthors. Still we think that there may be some hope that our modules 
may be usable in Lapidus' project. In order to demonstrate this point of view we have made an explicit construction of our module based on Theorem 3.2 for the unit interval and shown that the spectral triple reproduces the usual metric, the Dixmier trace induces a multiple of the usual Lebesgue integral and the growth of the number of eigenvalues $N(\Lambda)$, numerically bounded by $\Lambda$, is such that $N(\Lambda) / \Lambda$ is bounded, but without limit for $\Lambda \rightarrow \infty$. This very last result shows that, in general, spectral triples are not uniquely determined, even though they may be constructed by fairly reasonable algorithms. The Dirac operator of this example will not satisfy Weyl's asymptotic formula in the way that there exists no constant $c$ such that $N(\Lambda)-c \Lambda$ is of lower order than $\Lambda$. The reason for the failure has to be sought in the geometric growth of the numerical value of the eigenvalues of the constructed Dirac operator. The $n$ 'th eigenvalue, according to numerical size is roughly $2^{n}$ and its multiplicity is also $2^{n}$. Hence the gaps in the spectrum grow exponentially, but so do the multiplicities too, and that makes $N(\Lambda) / \Lambda$ a bounded function without a limit for $\Lambda \rightarrow \infty$.

In the book [4] Example 2a), p. 563, Connes introduces a spectral triple for a set consisting of just two points, and in [5] this construction is applied to a countable family of pairs of points from the classical middle third Cantor set in the unit interval. The direct sum of these two-dimensional modules and their Dirac operators gives a spectral triple for the algebra of continuous functions on the Cantor set, and it is possible to obtain a lot of exact geometric data for the Cantor set from this spectral triple. This construction has been studied in details and extended to certain fractal subsets of $\mathrm{R}^{n}$ by $\mathrm{D}$. Guido and T. Isola in [7], [8], [9]. Their constructions are based on the fact that many fractals in $\mathrm{R}^{n}$ can be obtained as limits for iterated function systems of contractions. As in Connes' construction they can then obtain a good description of the fractal in question by looking at a pair of points and the sequence of images of this pair, generated by the iterations of the function system. They also have another construction based on a single point. The pairs will then be of the form (child, parent) among the iterated images of the starting point.

In this paper we are studying an abstract compact metric space and we want to see to which extend it can be described using a spectral triple which is a countable direct sum of two-dimensional modules. We show that the metric can be recovered exactly from such a spectral triple. This construction shows that a metric is relatively easy to describe via a spectral triple, and that it is difficult to put a ranking to spectral triples for general compact metric spaces. The spectral triple which gives the metric back exactly is in principle optimal, because no information regarding the metric space is lost when we compare the algebra of continuous functions plus the spectral triple with the compact space plus the metric. On the other hand the spectral triple under consideration 
is not the right one because it is summable for any positive value. In general the spectral triple should somehow reflect the dimension of the space and the local density of the space, as it has been nicely pointed out in [8]. For this reason we give another construction of a spectral triple, which is much more complicated and does not give the metric back exactly, but a metric which is only Lipschitz equivalent, to the original one. This spectral triple reflects the local structures of the space and gives some computable estimates of the upper Minkowski dimension of the space. In concrete cases it is possible to do much better as shown by Guido and Isola [7], [8], [9]. We have tried our spectral triple on the unit interval and we get the expected results except for the growth of the eigenvalues of the Dirac operator. The number $N(\Lambda)$ of eigenvalues numerically dominated by $\Lambda$ is of the order $O(\Lambda)$, but $N(\Lambda) / \Lambda$ has no limit for $\Lambda \rightarrow \infty$. It is possible to obtain similar results for the unit cube in $R^{d}$, but the details for the unit interval are already many.

\section{Notation and definitions}

The paper deals with elementary aspects of metric spaces and uses some of the language of Connes theory of non commutative geometry to do so. The standard reference to Connes work is his book [4]. We will also use some basic results on operator algebras, and all what we use can be found in the books by Kadison and Ringrose [11], but many other text books on operator algebra will also describe the concepts we are using.

In the introduction we referred to Connes' construction of a spectral triple based on two points [4] p. 563, 2. Example a). We will use this construction quite a lot and call such a spectral triple a two-point spectral triple. The basic idea is that for a subset consisting of two different points $\{x, y\}$ of a compact metric space $(\mathscr{T}, d)$ it is possible to construct a spectral triple which can express the distance between these points. It is done via the following definition.

Definition 2.1. Let $(\mathscr{T}, d)$ denote a compact metric space and $x, y$ be points in $\mathscr{T}$. The two-point spectral triple $\mathrm{ST}_{x, y}:=\left(\mathrm{C}(\mathscr{T}), H_{x, y}, D_{x, y}\right)$ is defined by

(a) If $x=y$ then $H_{x, y}=\{0\}$.

(b) If $x \neq y$ then $H_{x, y}=\mathrm{C}^{2}$ and $\pi_{x, y}: \mathrm{C}(\mathscr{T}) \rightarrow B\left(H_{x, y}\right)$ a representation given by.

(i) for $f \in \mathrm{C}(\mathscr{T})$ and $(\alpha, \beta) \in H_{x, y} \pi_{x, y}(f)(\alpha, \beta):=(f(x) \alpha, f(y) \beta)$

(ii) $D_{x, y}: H_{x, y} \rightarrow H_{x, y}$ and $D_{x, y}(\alpha, \beta)=\left(\frac{\beta}{d(x, y)}, \frac{\alpha}{d(x, y)}\right)$.

We remark that for the metric space $(\mathscr{T}, d)$ it follows by an elementary calculation that the metric $d_{\mathrm{ST}_{x, y}}$ which by Definition 1.2 is induced on $\mathscr{T}$ will 
be given by the following table.

$$
d_{\mathrm{ST}_{x, y}}(u, v)= \begin{cases}0 & \text { if } u=v \\ d(x, y) & \text { if }\{u, v\}=\{x, y\} \\ \infty & \text { if } u \neq v \text { and }\{u, v\} \neq\{x, y\}\end{cases}
$$

We will use some ingredients of the theory of dimension of metric spaces. We will not repeat much of it, but refer the reader to the book by Falconer [6]. In order to use this language we will use the following notation.

Definition 2.2. Let $(\mathscr{T}, d)$ denote a metric space, then for $r \geq 0$ and $t \in \mathscr{T}$ we will let $\mathrm{B}(t, r)$ denote the closed ball of radius $r$ centered at $t$.

For a compact metric space $(\mathscr{T}, d)$ and a positive real $r$ the compactness implies that $\mathscr{T}$ can be covered by a finite number of closed balls of radius $r$. Such a covering will be called minimal if the number of balls is minimal among all finite coverings by closed balls of radius $r$. The minimal number in such a covering is denoted $N_{r}$ and we remind the reader of the following definition

Definition 2.3. The upper Minkowski dimension - or upper box counting dimension - is defined as

$$
\overline{\operatorname{dim}_{\mathrm{M}}}(\mathscr{T}):=\limsup _{r \rightarrow 0} \frac{\log \left(N_{r}\right)}{-\log (r)} .
$$

This supremum may not be finite, but if the set $\mathscr{T}$ is a subset of $\mathbf{R}^{d}$ and the metric on $\mathscr{T}$ is the one inherited from $\mathrm{R}^{d}$ then the upper Minkowski dimension is at most $d$.

\section{Sums of two-dimensional triples}

This section contains two examples of spectral triples associated to a compact metric space. Both triples are countable sums of two-dimensional modules and both induce metrics for the given compact topology. The first example gives the metric back exactly and the second triple depends on a parameter $\delta>0$, and it can be constructed such that the induced metric is within a $\delta$-distance of the original one. Then why bother with the second example. The reason is that the first triple is finitely summable for any positive real number $s$. Hence this spectral triple contains no information on the dimension of the space. The other spectral triple reflects dimension properties such as the upper Minkowski dimension. It is much harder to construct and not so precise with respect to the metric, but it is probably a more relevant module for topological investigations. 
One can then ask what is the difference. For us it seems that the explanation for this phenomenon has to be found in the fact that the module $H$, we are using, is a countable sum of two-dimensional Hilbert spaces. This means that the operators in the representation of the continuous functions on the compact metric space are all in a discrete maximal Abelian von Neumann subalgebra $\mathscr{A}$ of $B(H)$. If $D$ is a Dirac operator for the module, and $N$ is a self-adjoint operator affiliated with $\mathscr{A}$, such that $N+D$ has a self-adjoint closure, then for any operator $a \in \mathscr{A}$ we have $[D, a]=[D+N, a]$. Now such an $N$ can be chosen relatively freely and such that $\left(I+(D+N)^{2}\right)^{-s / 2}$ is of trace class for any positive $s$.

THEOREM 3.1. Let $(\mathscr{T}, d)$ denote a compact metric space, then there exists a sequence of pairs of non-equal points $\left\{x_{n}, y_{n}\right\}$ from $\mathscr{T}$ such that the sum of all the two-point spectral triples $\mathrm{ST}_{x_{n}, y_{n}}$ becomes a spectral triple for $\mathrm{C}(\mathscr{T})$. This triple is denoted $\mathrm{ST}(d)$, it induces the given metric $d$ and it is summable for any positive real $s$.

Proof. Since $\mathscr{T}$ is compact and metric it contains a dense sequence $\left(t_{i}\right)$. We can then let the sequence $\left\{x_{n}, y_{n}\right\}$ be a numbering of the set of unordered pairs $\left\{\left\{t_{i}, t_{j}\right\} \mid i, j \in \mathbf{N}\right.$ and $\left.t_{i} \neq t_{j}\right\}$. For each pair $\left\{x_{n}, y_{n}\right\}$ we consider the two-point module $\mathrm{ST}_{x_{n}, y_{n}}$, but we will modify $D_{x_{n}, y_{n}}$ such that new Dirac operator, which we call $D_{n}$ is given by the following 2 by 2 matrix

$$
D_{n}:=\left(\begin{array}{cc}
2^{n} & \frac{1}{d\left(x_{n}, y_{n}\right)} \\
\frac{1}{d\left(x_{n}, y_{n}\right)} & -2^{n}
\end{array}\right)
$$

The other items are fixed, but in order to ease the notation, we will let $H_{n}$ denote the two-dimensional Hilbert space $H_{x_{n}, y_{n}}$ and $\pi_{n}$ is the representation $\pi_{x_{n}, y_{n}}$ of $\mathrm{C}(\mathscr{T})$ on $H_{n}$. It is easy to see that

$$
\forall f \in \mathrm{C}(\mathscr{T}):\left[D_{n}, \pi_{n}(f)\right]=\left(\begin{array}{cc}
0 & \frac{f\left(y_{n}\right)-f\left(x_{n}\right)}{d\left(x_{n}, y_{n}\right)} \\
\frac{f\left(x_{n}\right)-f\left(y_{n}\right)}{d\left(x_{n}, y_{n}\right)} & 0
\end{array}\right) .
$$

Let us start by showing that there exists a dense set of functions $f$ in $\mathrm{C}(\mathscr{T})$ such that the commutators $[D, \pi(f)]$ are all bounded and densely defined. To this end we define

$$
\mathcal{N}:=\{f \in \mathrm{C}(\mathscr{T}) \mid \exists y \in \mathscr{T} \forall x \in \mathscr{T} f(x):=d(x, y)\}
$$

It is a simple consequence of the triangle inequality that for any of the pairs 
$\left\{x_{n}, y_{n}\right\}$ and for any $f(u):=d(u, v)$ in $\mathcal{N}$ we have

$$
\left\|\left[D_{n}, \pi_{n}(f)\right]\right\|=\frac{\left|d\left(x_{n}, v\right)-d\left(y_{n}, v\right)\right|}{d\left(x_{n}, y_{n}\right)} \leq 1
$$

Hence for each $f$ in $\mathcal{N}$ we have $\|[D, \pi(f)]\| \leq 1$ and since this operator clearly is defined on the linear span of the $H_{n}$ spaces, it is densely defined too. Since for any two points $u \neq v$ we have $d(u, v)>0=d(v, v)$, we see that the algebra generated by the functions in $\mathscr{N}$ and the constant function $I$ separates the points in $\mathscr{T}$, and hence by Stone-Weierstrass' theorem it is uniformly dense. On the other hand it follows from the derivation property of a commutator that all elements in this algebra have bounded commutators with $D$, so the first condition for $\mathrm{ST}(d)$ to be a spectral triple is fulfilled. Before we start to prove that the second condition holds, we will collect some of the results above for later use in the following statement.

(1) Let $\mathscr{M}:=\{g \in \mathrm{C}(\mathscr{T}) \mid\|[D, \pi(g)]\| \leq 1\}$ then $\mathscr{M}$ separates the states.

Let us then return to the definitions of the $D_{n}$ operators and of $D$. First we compute the eigenvalues of each $D_{n}$, and we get the set below.

$$
\sigma\left(D_{n}\right)=\left\{-\sqrt{2^{2 n}+d\left(x_{n}, y_{n}\right)^{-2}}, \sqrt{2^{2 n}+d\left(x_{n}, y_{n}\right)^{-2}}\right\}
$$

So the two eigenvalues are both numerically bigger than $2^{n}$ and we can conclude that for the operator $\left(I+D^{2}\right)^{-1}$ there are at most $2 n$ eigenvalues of absolute value bigger than $2^{-n}$. This means that $\left(I+D^{2}\right)^{-1}$ is compact and that for any positive real number $s$ we have

$$
\operatorname{tr}\left(\left(I+D^{2}\right)^{-s / 2}\right)<2 \sum_{n=1}^{\infty} 2^{-n s}<\infty .
$$

It then follows that $(\mathrm{C}(\mathscr{T}), H, D)$ is a spectral triple which is summable for any positive $s$.

We will now show that the metric, say $d_{\mathrm{ST}(d)}$ induced by $\mathrm{ST}(d)$ on the state space has the property that it agrees with the original metric on the pure states, i.e. the point measures. We will first show that for any pair of points $\{x, y\}$ from $\mathscr{T}$ we have $d(x, y) \leq d_{\mathrm{ST}(d)}(x, y)$. This type of inequality is quite general and follows from the fact that the set of functions $\mathcal{N}$ is a subset of the set $\mathscr{M}$. To be more specific, let $s, t$ be given points in $\mathscr{T}$ and define the function $g(u):=d(u, t)$ in $\mathcal{N}$, then

$$
\begin{aligned}
d_{\mathrm{ST}(d)}(s, t) & =\sup _{f \in \mathcal{M}}|f(s)-f(t)| \geq \sup _{f \in \mathcal{N}}|f(s)-f(t)| \\
& \geq|g(s)-g(t)|=|d(s, t)-d(t, t)|=d(s, t) .
\end{aligned}
$$


Let us then show the inequality $d_{\mathrm{ST}(d)}(s, t) \leq d(s, t)$. Let again $s, t$ be given from $\mathscr{T}$, and let us suppose first that

$$
d_{\mathrm{ST}(d)}(s, t)>\operatorname{diam}(\mathscr{T}):=\sup \{d(u, v) \mid u, v \in \mathscr{T}\} .
$$

Then there must exist a function $f \in \mathcal{M}$ such that $|f(s)-f(t)|>\operatorname{diam}(\mathscr{T})$. Let then $\varepsilon>0$ be chosen such that $|f(s)-f(t)|-\operatorname{diam}(\mathscr{T})>3 \varepsilon$, and find a natural number $n$ such that the pair $\left(x_{n}, y_{n}\right)$ is so close to the pair $(s, t)$ that $\left|f(s)-f\left(x_{n}\right)\right|<\varepsilon$ and $\left|f(t)-f\left(y_{n}\right)\right|<\varepsilon$. Since $f \in \mathcal{M}$ we have $\left|f\left(x_{n}\right)-f\left(y_{n}\right)\right| \leq d\left(x_{n}, y_{n}\right) \leq \operatorname{diam}(\mathscr{T})$ and we get the contradiction

$$
\operatorname{diam}(\mathscr{T})+3 \varepsilon<|f(s)-f(t)| \leq\left|f\left(x_{n}\right)-f\left(y_{n}\right)\right|+2 \varepsilon \leq \operatorname{diam}(\mathscr{T})+2 \varepsilon
$$

We now know that $d_{\mathrm{ST}(d)}(s, t) \leq \operatorname{diam}(\mathscr{T})<\infty$, so for any positive $\varepsilon$ there exists a function $f \in \mathcal{M}$ such that $|f(s)-f(t)|>d_{\mathrm{ST}(d)}(s, t)-\varepsilon / 5$. Again, using the continuity of $f$ and the original metric $d$, we find a pair $\left(x_{n}, y_{n}\right)$ from the sequence upon which the spectral triple is build, such that $\left|f(s)-f\left(x_{n}\right)\right|<$ $\varepsilon / 5,\left|f(t)-f\left(y_{n}\right)\right|<\varepsilon / 5, d\left(s, x_{n}\right)<\varepsilon / 5$ and $d\left(t, y_{n}\right)<\varepsilon / 5$. Having this we finally may conclude as follows

$$
\begin{aligned}
d_{\mathrm{ST}(d)}(s, t) & <|f(s)-f(t)|+\varepsilon / 5 \\
& <\left|f\left(x_{n}\right)-f\left(y_{n}\right)\right|+3 \varepsilon / 5 \\
& \leq d\left(x_{n}, y_{n}\right)+3 \varepsilon / 5 \\
& <d(s, t)+\varepsilon .
\end{aligned}
$$

So for any pair $(s, t)$ of points from $\mathscr{T}$ we have $d(s, t)=d_{\mathrm{ST}(d)}(s, t)$. We are nearly done, but we think it is appropriate to note that the metric $d_{\mathrm{ST}(d)}$ really is a metric on the state space and that it generates the $w^{*}$-topology on that space. This can be seen in many ways. We will use the method which Rieffel has described in [22] and we have used in [1], Proposition 3.2. Consider again the set $\mathcal{M}$, fix an element $v \in \mathscr{T}$ and define a subset $\mathcal{M}_{v}$ of $\mathcal{M}$ by

$$
\mathcal{M}_{v}:=\{f \in \mathcal{M} \mid f(v)=0\}
$$

We know already by (1) that $\mathcal{M}$ separates the states of $\mathrm{C}(\mathscr{T})$, so according to Rieffel we then just have to show that $\mathscr{M}_{v}$ is relatively compact in $\mathrm{C}(\mathscr{T})$. This follows from the construction of $\mathscr{M}_{v}$, since by the definition we have for any $f \in \mathcal{M}_{v}$ and any pair of points $\{s, t\}$ from $\mathscr{T}$ that $|f(s)-f(t)| \leq d_{\mathrm{ST}(d)}(s, t)=$ $d(s, t)$. This means that the functions in $\mathcal{M}$ are equicontinuous. Further for any $t \in \mathscr{T}$ and any $f \in \mathscr{M}_{v}$ we have $|f(t)|=|f(t)-f(v)| \leq d(t, v) \leq \operatorname{diam}(\mathscr{T})$, so the set $\mathscr{M}_{v}$ is also bounded. By Arzelà-Ascoli's Theorem we get that $\mathscr{M}_{v}$ is a relatively compact subset of $\mathrm{C}(\mathscr{T})$ and the theorem follows. 
THeORem 3.2. Let $(\mathscr{T}, d)$ denote a compact metric space and let $0<\delta$ be a real number. Then there exists a countable set $\mathscr{J}$ of pairs of non-equal points $\{x, y\}$ from $\mathscr{T}$ such that the sum of all the two-point spectral triples $\mathrm{ST}_{x, y}$ over $\{x, y\} \in \mathscr{J}$ becomes a spectral triple for $\mathrm{C}(\mathscr{T})$. This triple is denoted $\mathrm{ST}(\delta)$ and it induces a metric $d_{\delta}$ on $\mathscr{T}$ such that

$$
\forall s, t \in \mathscr{T}: d(s, t) \leq d_{\delta}(s, t) \leq(1+\delta) d(s, t)
$$

If the upper Minkowski dimension $\overline{\operatorname{dim}_{\mathrm{M}}}(\mathscr{T})$ is finite then the module is finitely summable for any real $s$ such that $s>2 \overline{\operatorname{dim}_{M}}(\mathscr{T})$.

If the module is summable for some $s>0$ and the topological space $\mathscr{T}$ is connected, then $\overline{\operatorname{dim}_{\mathrm{M}}}(\mathscr{T})$ is at most $s$.

Proof. The construction can be done in many ways and it may be reasonable to give a proof which reflects this. In this way the proof becomes a little less transparent, but more easily applicable to different metric sets of fractal type. The proof is based on a sequence of positive numbers $\left(r_{n}\right), r_{n}=\theta \rho^{n-1}$ for some strictly positive real $\theta$ and a real $\rho$ such that $0<\rho<1$. The simplest argument for the general case can probably be obtained when $\theta=\operatorname{diam}(\mathscr{T})$, the diameter of $\mathscr{T}$, and $\rho=1 / 2$, but if one looks at the usual middle-third Cantor set it turns out that $\theta=1 / 2$ and $\rho=1 / 3$ will be the most natural choice. On the other hand it is quite clear that other sequences $\left(r_{n}\right)$ of positive reals which are finitely summable may be used in certain cases. We have not been able to find a proof which works in this generality, so the proof here will be based on sequences of geometric descent.

Let us then suppose that some positive reals $\delta, \theta$ and $0<\rho<1$ are given. We are then going to specify a sequence $\left(\mathscr{T}_{n}\right)$ of finite subsets of the set $\mathscr{T}$, on which we can base our constructions. The points in $\mathscr{T}_{n}$ will consist of the centers of a minimal covering of $\mathscr{T}$ by closed balls of radius $\theta \rho^{n-1}$. It should be remarked that each set $\mathscr{T}_{n}$ is not uniquely determined and it is also possible that two points $s_{m}, t_{n}$ from the sets $\mathscr{T}_{m}$ and $\mathscr{T}_{n}$ for $m \neq n$ may be equal. This will not cause any trouble as one can see below. Later the numbers $\delta, \theta$ and $\rho$ will determine which pairs of centers from the union $\cup \mathscr{T}_{n}$ we will use for the construction of the two-point spectral triples, which will be the summands in the spectral triple $\mathrm{ST}(\delta)$.

Given $\delta$, then the smaller it is, the more accurate the metric $d_{\delta}$ describes the original metric. A better approximation to the given $d$ can only be achieved at a cost of having more points in the model. The exact content of this statement is coded in the integer $l(\delta)$ which we define below. We use the term interaction length for $l(\delta)$. It is not possible to see the meaning of this number right away, but it follows - hopefully - as the proof proceeds. The diameter of the space is given as $\operatorname{diam}(\mathscr{T})=\max \{d(s, t) \mid s, t \in \mathscr{T}\}$ and its logarithm plays a role 
for the size of $l(\delta)$ so we define an integer $k_{0}$ - not necessarily non negative by

$$
k_{0} \in \mathbf{Z}, \quad \theta \rho^{k_{0}+1}<\operatorname{diam}(\mathscr{T}) \leq \theta \rho^{k_{0}} .
$$

Then we can define the interaction length $l:=l(\delta)$ by the conditions:

$$
\begin{aligned}
& \text { If } \frac{4}{1-\rho}<\delta \text { then } l=\max \left\{0,-k_{0}\right\} \\
& \text { If } \frac{4}{1-\rho} \geq \delta \text { then } l \in \mathbf{N} \text { is the least natural number s. t. } \\
& l \geq-k_{0} \text { and } \frac{4 \rho^{l}}{1-\rho}<\delta .
\end{aligned}
$$

Remark that even though $k_{0}$ may be negative the inequalities above imply that

$$
k_{0}+l \geq 0 \quad \text { and } \quad \frac{4 \rho^{l}}{1-\rho}<\delta,
$$

which will be useful later. The two-point modules which will go into the construction of the spectral triple $\mathrm{ST}(\delta)$ can then be determined. As index set $\mathscr{J}$ we will consider all pairs of points $\{x, y\}$ from $\mathscr{T}$ such that

(i) $\exists n \in \mathrm{N}: x \in \mathscr{T}_{n}$ and $y \in \mathscr{T}_{n} \cup \mathscr{T}_{n+1}$.

(ii) $x \neq y$.

(iii) If $y \in \mathscr{T}_{n}$ then $d(x, y) \leq\left(2+\rho^{-(l+1)}\right) \theta \rho^{n-1}$

(iv) If $y \in \mathscr{T}_{n+1}$ then $d(x, y) \leq(1+\rho) \theta \rho^{n-1}$.

Now our first task is to show that the direct sum of all these two-point spectral triples will give a spectral triple. The first part of the proof of this is done as in the proof of Theorem 3.1, but we remind the reader that we are now using the standard two-point spectral triples as defined in Definition 2.1. We will let $H$ denote the Hilbert space sum of all the $H_{x, y}$ for all the pairs $\{x, y\} \in \mathscr{J}$. This sum is countable and we may then define a self-adjoint operator $D$ on $H$ as the closure of the sum of all the operators $D_{x, y}$ on $H_{x, y}$ over $\{x, y\} \in \mathscr{J}$. We will let $\pi$ denote the representation of $\mathrm{C}(\mathscr{T})$ on $H$ which is equal to the sum of the representations $\pi_{x, y}$ on the spaces $H_{x, y}$, for $\{x, y\} \in \mathscr{J}$.

As in the proof of Theorem 3.1 we define the set $\mathcal{N}$, by

$$
\mathscr{N}:=\{f \in \mathrm{C}(\mathscr{T}) \mid \exists y \in \mathscr{T} \forall x \in \mathscr{T}: f(x)=d(x, y)\} .
$$

and you will find, as before, that the algebra generated by $\mathscr{N}$ and the unit $I$ is uniformly dense in $\mathrm{C}(\mathscr{T})$, and for any element, $f$ in this algebra, the 
commutator $[D, \pi(f)]$ is bounded. The first condition in Definition 1.1 is then fulfilled. The second condition, which asks for the compactness of the operator $\left(I+D^{2}\right)^{-1}$ has to be considered in more details in order to be verified. In order to prove that $\left(I+D^{2}\right)^{-1}$ is compact we just have to show that for any positive number $r$ the self-adjoint operator $D$ has only finitely many eigenvalues in the interval $[-r, r]$. Let $\{x, y\}$ be a pair from $\mathscr{J}$ then the eigenvalues, i.e. the spectrum $\sigma\left(D_{x, y}\right)$ of $D_{x, y}$ is the set $\left\{-d(x, y)^{-1}, d(x, y)^{-1}\right\}$, so we get that

$$
\begin{aligned}
& \text { If } x, y \in \mathscr{T}_{n},\{x, y\} \in \mathscr{J} \text { and } \lambda \in \sigma\left(D_{x, y}\right) \text { then }|\lambda| \geq \frac{\rho^{1-n}}{\left(2+\rho^{-(l+1)}\right) \theta} . \\
& \text { If } x \in \mathscr{T}_{n}, y \in \mathscr{T}_{n+1},\{x, y\} \in \mathscr{J} \text { and } \lambda \in \sigma\left(D_{x, y}\right) \text { then }|\lambda| \geq \frac{\rho^{1-n}}{(1+\rho) \theta} .
\end{aligned}
$$

In both of the cases we see, that for any given $r>0$ there will be an $n_{0}$ such that only the finitely many elements $\{x, y\} \in \mathscr{J}$ for which $x \in \mathscr{T}_{n}$ for some $n \leq n_{0}$ can yield operators $D_{x, y}$ with eigenvalues of absolute value less than or equal to $r$.

We will now turn to the properties of the metric induced by $\operatorname{ST}(\delta)$. The first inequality claimed, $d(x, y) \leq d_{\delta}(x, y)$, is quite general and it is proved exactly as in the proof of Theorem 3.1

We will then show the inequality $d_{\delta}(s, t) \leq(1+\delta) d(s, t)$. Let $s, t$ be given from $\mathscr{T}$, then we are first going to determine the scale of the argument for this particular pair $(s, t)$, and this is done by finding the unique integer $k$, it may be negative, such that

$$
\theta \rho^{k}<d(s, t) \leq \theta \rho^{k-1} .
$$

By definition of $k_{0}$ we find that $k>k_{0}$ and by equation (7) we then get

$$
k+l \geq 1
$$

For any natural number $n$ we can by equation (10) choose points $a_{n}, b_{n} \in$ $\mathscr{T}_{k+l+n}$ such that

$$
d\left(s, a_{n}\right) \leq \theta \rho^{(k+l+n-1)} \quad \text { and } \quad d\left(t, b_{n}\right) \leq \theta \rho^{(k+l+n-1)} .
$$

Based on this we get via the triangle inequality

$$
\begin{aligned}
\forall n \in \mathrm{N}: d\left(a_{n+1}, a_{n}\right) & \leq \theta \rho^{(k+l+n)}+\theta \rho^{(k+l+n-1)} \quad \text { and } \\
d\left(b_{n+1}, b_{n}\right) & \leq \theta \rho^{(k+l+n)}+\theta \rho^{(k+l+n-1)} . \\
d\left(a_{1}, b_{1}\right) & \leq d(s, t)+2 \theta \rho^{k+l} .
\end{aligned}
$$


From the last one and the definition of $k$ we get

$$
d\left(a_{1}, b_{1}\right) \leq \theta \rho^{k-1}+2 \theta \rho^{k+l}=\theta \rho^{k+l}\left(2+\rho^{-(l+1)}\right) .
$$

Since both $a_{1}$ and $b_{1}$ belongs to $\mathscr{T}_{k+l+1}$ the last inequality implies that the pair $\left\{a_{1}, b_{1}\right\}$ is in $\mathscr{J}$ and the two-point spectral triple $\mathrm{ST}_{a_{1}, b_{1}}$ is a summand in the spectral triple we consider.

Next we are going to show that the pairs $\left\{a_{n}, a_{n+1}\right\},\left\{b_{n}, b_{n+1}\right\}$ also belong to $\mathscr{J}$. Let us look at $\left\{a_{n}, a_{n+1}\right\}$ then $a_{n} \in \mathscr{T}_{k+l+n}$ and $a_{n+1} \in \mathscr{T}_{k+l+n+1}$, so we must check their distance. According to (12) we get

$$
d\left(a_{n+1}, a_{n}\right) \leq \theta \rho^{(k+l+n)}+\theta \rho^{(k+l+n-1)}=\theta \rho^{(k+l+n-1)}(1+\rho),
$$

and it follows that these pairs also go into the formation of $\operatorname{ST}(\delta)$, and analogously the pairs $\left\{b_{n}, b_{n+1}\right\}$ belong to $\mathscr{J}$ too.

We can then collect the estimates. The basic idea is that we jump from $s$ to an $a_{n}$, which is nearby, and then continues to jump from $a_{i}$ to $a_{i-1}$ until we reach $a_{1}$. From here we jump to $b_{1}$ and continues the jumping up to $b_{n}$ from where there is only a short distance to $t$. All the jumps except the first and the last are controlled by $\operatorname{ST}(\delta)$. The end jumps can be made of arbitrary small importance by continuity arguments. To be precise let us choose a function $f \in \mathcal{M}$, i.e. a continuous function such that $\|[D, \pi(f)]\| \leq 1$ and try to get an upper estimate for $|f(s)-f(t)|$. For a given $\varepsilon>0$ there exists an $n \in \mathbf{N}$ such that $\left|f(s)-f\left(a_{n}\right)\right| \leq \varepsilon / 2$ and $\left|f(t)-f\left(b_{n}\right)\right| \leq \varepsilon / 2$. By construction the function $f \in \mathscr{M}$ has the property that for any pair $\{x, y\} \in \mathscr{J}$ we have $|f(x)-f(y)| \leq d(x, y)$, so

$$
\begin{aligned}
\mid f(s) & -f(t)|\leq| f\left(a_{n}\right)-f\left(b_{n}\right) \mid+\varepsilon \\
& \leq\left|f\left(a_{1}\right)-f\left(b_{1}\right)\right|+\sum_{i=1}^{n-1}\left(\left|f\left(a_{i}\right)-f\left(a_{i+1}\right)\right|+\left|f\left(b_{i}\right)-f\left(b_{i+1}\right)\right|\right)+\varepsilon \\
& \leq d\left(a_{1}, b_{1}\right)+\sum_{i=1}^{n-1}\left(d\left(a_{i}, a_{i+1}\right)+d\left(b_{i}, b_{i+1}\right)\right)+\varepsilon \\
& \leq d(s, t)+4 \theta \sum_{i=1}^{\infty} \rho^{(k+l-1+i)}+\varepsilon \\
& =d(s, t)+\frac{4 \theta \rho^{k+l}}{1-\rho}+\varepsilon \quad \text { and by (9) } \\
& \leq d(s, t)\left(1+\frac{4 \rho^{l}}{1-\rho}\right)+\varepsilon \quad \text { which by (7) } \\
& \leq d(s, t)(1+\delta)+\varepsilon .
\end{aligned}
$$


Hence $d_{\delta}(x, y) \leq(1+\delta) d(s, t)$.

We will then prove that $d_{\delta}$ generates the $w^{*}$-topology on the state space $\mathscr{S}(\mathrm{C}(\mathscr{T}))$. This is also done as in the proof of Theorem 3.1 so we have to show that the set $\mathscr{M}_{v}$, which is defined as in the proof of Theorem 3.1 in the relations (1) and (2) is relatively compact and separates the states of $\mathrm{C}(\mathscr{T})$. The proof here is practically the same as the one given at the end of the proof of Theorem 3.1. The only difference being that we get the equicontinuity and the boundedness of $\mathscr{M}_{v}$ from the following inequality.

$$
\forall f \in \mathscr{M}_{v} \forall s, t \in \mathscr{T}:|f(s)-f(t)| \leq(1+\delta) d(s, t) .
$$

We will now turn to the summability questions. We think that the results obtainable will turn out to be much more precise in the future, but we have gotten into a lot of combinatorial problems when we tried to sharpen our results on the connection between finite summability of ST $(\delta)$ and finiteness of the upper Minkowski dimension of $\mathscr{T}$.

Let us suppose that the upper Minkowski dimension, $\overline{\operatorname{dim}_{M}}(\mathscr{T})$ is finite, and recall that for $n \in \mathrm{N}$ the set $\mathscr{T}_{n}$ consists of the centers of a minimal covering of $\mathscr{T}$ by closed balls of radius $\theta \rho^{n-1}$. We will let $\left|\mathscr{T}_{n}\right|$ denote the number of points in $\mathscr{T}_{n}$ then we get by Definition 2.3

$$
\overline{\operatorname{dim}_{\mathrm{M}}}(\mathscr{T}):=\limsup _{n \rightarrow \infty} \frac{\log \left(\left|\mathscr{T}_{n}\right|\right)}{-\log \left(\theta \rho^{n-1}\right)}<\infty .
$$

Let $\mu$ be a real number such that $\mu>\overline{\operatorname{dim}_{\mathrm{M}}}(\mathscr{T})$ then there must exist a natural number $n_{0}$ such that

$$
\forall n>n_{0}: \frac{\log \left(\left|\mathscr{T}_{n}\right|\right)}{-\log \left(\theta \rho^{n-1}\right)}<\mu,
$$

and then there must be a natural number $n_{1} \geq n_{0}$ such that

$$
\forall n>n_{1}: \frac{\log \left(\left|\mathscr{T}_{n}\right|\right)}{-\log \left(\rho^{n}\right)}<\mu,
$$

which implies that

$$
\forall n>n_{1}:\left|\mathscr{T}_{n}\right|<\rho^{-\mu n} .
$$

We can now make estimates of the value of $\operatorname{tr}\left(\left(I+D^{2}\right)^{-s / 2}\right)$ and we will show that it is finite for any real $s>2 \overline{\operatorname{dim}_{\mathrm{M}}}(\mathscr{T})$. Suppose such an $s$ is given and $\mu$ is chosen such that $2 \overline{\operatorname{dim}_{\mathrm{M}}}(\mathscr{T})<2 \mu<s$. First we remark that for $\lambda \neq 0$ we have the inequality

$$
\frac{1}{\sqrt{1+|\lambda|^{2}}} \leq|\lambda|^{-1}
$$


When we have to estimate the trace $\operatorname{tr}\left(\left(I+D^{2}\right)^{-s / 2}\right)$ we must go back to the definition of $D$, and we find that the index set $\mathscr{J}$, over which $D$ is formed, is grouped into disjoint subsets $\mathscr{J}_{n}, n \in \mathrm{N}$ such that

$$
\mathscr{J}_{n}:=\left\{\{x, y\} \in \mathscr{J} \mid x \in \mathscr{T}_{n} \text { and } y \in \mathscr{T}_{n} \cup \mathscr{T}_{n+1}\right\} .
$$

Having this and the inequality just above we get

$$
\operatorname{tr}\left(\left(I+D^{2}\right)^{-s / 2}\right) \leq \sum_{n=1}^{\infty}\left(\sum_{\{x, y\} \in \mathscr{Z}_{n}} 2 d(x, y)^{s}\right) .
$$

In this sum we recall that there are upper limits for $d(x, y)$ which were used in the definition of the spectral triple. By inspection of the definition it turns out that we have the following inequalities

$$
\exists c>0 \forall n \in \mathbf{N} \forall\{x, y\} \in \mathscr{J}_{n}: d(x, y) \leq c \rho^{n} .
$$

The number, say $\left|\mathscr{J}_{n}\right|$, of pairs in $\mathscr{J}_{n}$ is not known, because it depends of the local structure of the metric $d$. Without further knowledge we can only get some very rough estimates of the size of $\left|\mathscr{J}_{n}\right|$, but we can remark that for a nice subset of the space $\mathrm{R}^{d}$ such as the unit cube, one can do much better. The general estimate we can get is simply the largest possible numbers of pairs, so we get

$$
\forall n \in \mathrm{N}: \quad\left|\mathscr{J}_{n}\right| \leq \frac{\left|\mathscr{T}_{n}\right|^{2}}{2}+\left|\mathscr{T}_{n}\right|\left|\mathscr{T}_{n+1}\right|
$$

This can then be combined with the estimates $\left|\mathscr{T}_{n}\right|<\rho^{-\mu n}$, for $n>n_{1}$, so we obtain

$$
\begin{aligned}
& \operatorname{tr}\left(\left(I+D^{2}\right)^{-s / 2}\right) \\
& \leq \sum_{n=1}^{n_{1}}\left(\sum_{\{x, y\} \in \mathscr{J}_{n}} 2 d(x, y)^{s}\right)+\sum_{n>n_{1}}\left(\left(c \rho^{n}\right)^{s}\left(\frac{\rho^{-2 \mu n}}{2}+\rho^{-\mu(2 n+1)}\right)\right) .
\end{aligned}
$$

This means that there exist positive reals $A$ and $B$ such that

$$
\operatorname{tr}\left(\left(I+D^{2}\right)^{-s / 2}\right) \leq A+B \sum_{n>n_{1}} \rho^{(s-2 \mu) n}<\infty,
$$

and the module is finitely summable for any $s>2 \overline{\operatorname{dim}_{M}}(\mathscr{T})$.

Let us now suppose that the module is finitely summable for some $s>0$ and the space $\mathscr{T}$ is connected. By the construction of the spectral triple (8) a pair of centers $\{x, y\}$ in $\mathscr{T}_{n}$ belongs to $\mathscr{J}_{n}$ if $d(x, y) \leq\left(2+\rho^{-(l+1)}\right) \theta \rho^{n-1}$. This means, among other things, that there exists a natural number $n_{2}$ such that 
for any natural number $n>n_{2}$ and any pair $\{x, y\} \in \mathscr{T}_{n}$ such that $x, y \in \mathscr{J}_{n}$ then we have

$$
d(x, y) \leq 1, \quad \text { and } \quad 2 \theta \rho^{n-1} \leq \operatorname{diam}(\mathscr{T}) / 3 .
$$

The inequality $d(x, y) \leq 1$ implies $\left(1+d(x, y)^{-2}\right)^{-s / 2} \geq 2^{-s / 2} d(x, y)^{s}$, so the assumption of finite summability of the module and these considerations imply

$$
\begin{aligned}
\infty & >\operatorname{tr}\left(\left(I+D^{2}\right)^{-s / 2}\right) \\
& =\sum_{n=1}^{\infty}\left(\sum_{\{x, y\} \in \mathscr{I}_{n}} 2\left(1+d(x, y)^{-2}\right)^{-s / 2}\right) \\
& \geq \sum_{n>n_{2}}\left(\sum_{\{x, y\} \in \mathscr{F}_{n}, x, y \in \mathscr{T}_{n}} 2^{(1-s / 2)} d(x, y)^{s}\right) .
\end{aligned}
$$

We are now going to show that for each $n>n_{2}$ there are at least $\left|\mathscr{T}_{n}\right| / 2$ number of pairs $\{x, y\}$ in $\mathscr{J}_{n}$ such that $x$ and $y$ both are in $\mathscr{T}_{n}$ and $d(x, y) \geq \theta \rho^{n-1}$. We do this by showing that for any $x \in \mathscr{T}_{n}$ there exists at least one $y \in \mathscr{T}_{n}$ such that $\{x, y\} \in \mathscr{J}_{n}$ and $d(x, y) \geq \theta \rho^{n-1}$. Let $x \in \mathscr{T}_{n}$ then there must exist a $t \in \mathscr{T}$ such that $d(x, t) \geq \frac{\operatorname{diam}(\mathscr{T})}{3}$. By assumptions $\mathscr{T}$ is connected and for $n>n_{2}, 2 \theta \rho^{n-1} \leq \frac{\operatorname{diam}(\mathscr{T})}{3}$, hence there must be a $u \in \mathscr{T}$ such that $d(x, u)=2 \theta \rho^{n-1}$. Finally we choose $y \in \mathscr{T}_{n}$ such that $d(y, u) \leq \theta \rho^{n-1}$ and we get

$$
d(x, y) \leq d(x, u)+d(u, y) \leq 3 \theta \rho^{n-1}<\left(2+\rho^{-(l+1)}\right) \theta \rho^{n-1},
$$

so by (8) the pair $\{x, y\}$ belongs to the index set $\mathscr{J}_{n}$, if $x \neq y$. On the other hand, if we use the triangle inequality the other way around, we get

$$
d(x, y) \geq d(x, u)-d(u, y) \geq \theta \rho^{n-1}>0, \quad \text { so } \quad x \neq y .
$$

We have then seen that any point $x$ in $\mathscr{T}_{n}$ belongs to a pair $\{x, y\}$ in $\mathscr{J}_{n}$ with $y$ in $\mathscr{T}_{n}$ and $d(x, y) \geq \theta \rho^{n-1}$, so we may continue our lower bound estimates (19) on the trace of $\left(I+D^{2}\right)^{-s / 2}$ in order to get the following result

$$
\sum_{n>n_{2}}\left|\mathscr{T}_{n}\right|\left(\rho^{s}\right)^{n}<\infty
$$

In particular this means that there exists a natural number $n_{3}>n_{2}$ such that 
for any $n>n_{3}$

$$
\begin{aligned}
\left|\mathscr{T}_{n}\right|\left(\rho^{s}\right)^{n} & <1, \quad \text { and } \\
\log \left|\mathscr{T}_{n}\right| & <-s \log \rho^{n} \\
\frac{\log \left|\mathscr{T}_{n}\right|}{-\log \rho^{n}} & <s, \quad \text { and for } n \text { suff. large } \\
\frac{\log \left|\mathscr{T}_{n}\right|}{-\log \left(\theta \rho^{n-1}\right)} & <s .
\end{aligned}
$$

Since the set $\mathscr{T}_{n}$ is a set of centers for a minimal covering of $\mathscr{T}$ by balls of radius $\theta \rho^{n-1}$, the last inequality shows that the upper Minkowski dimension of $\mathscr{T}$ is at most s, and the theorem follows.

REMARK 3.3. The result raises a number of questions. First of all, is it possible to construct discrete spectral triples which are more accurate with respect to the calculation of the Minkowski dimension? We think that the answer definitely is yes, and for all the known examples we have thought of, there are ad hoc constructions which are very precise. The works by Guido and Isola [7], [8], [9] offer spectral triples which works for a large collection of fractals in $\mathrm{R}^{n}$. Some of the problems we have faced when we have tried to get more precise results is - in the language of the proof just above - to determine the number of pairs $\{x, y\} \in \mathscr{J}_{n}$ which contains a given $x$. In known examples there exists a constant $c$ such that this number is at most $c$ for any value of $n$. In our proof of Theorem 3.2 the estimate is $3 / 2\left|\mathscr{T}_{n}\right|^{2}$ which is far away from being universally bounded. To illustrate the exact content of these cryptical remarks we will compute an example based on the unit interval. A similar result can be obtained for any unit cube in $\mathrm{R}^{d}$, but the counting of the multiplicities of the eigenvalues is much more complicated in higher dimensions, so in order to avoid huge combinatorial arguments we will just compute the Dirac operator $D$ for the unit interval based on the construction given in the proof of Theorem 3.2. We then show that this spectral triple behaves very nicely. The only un-excepted result is that for this module the so called Weyl's asymptotic formula for the growth of the eigenvalues does not hold completely. The reason for this is that this Dirac operator has gaps between the eigenvalues which grow exponentially and also the multiplicities of the eigenvalues grow exponentially. You can see the details just below.

EXAMPLE 3.4. We consider the unit interval $[0,1]$ with the usual metric. In the construction from the proof of Theorem 3.2 we let $\delta=9, \theta=1$ and $\rho=1 / 2$. Then for the spectral triple $\mathrm{ST}(9)=(\mathrm{C}([0,1]), H, D)$ constructed 
by the algorithm of Theorem 3.2 we get:

(a) The metric induced by the spectral triple is the usual one.

(b) The module is finitely summable for $s>1$, but not for $s=1$.

(c) Let for $\Lambda>0, N(\Lambda)$ denote the number of eigenvalues of $D$ of absolute value at most $\Lambda$ then

$$
10<\liminf _{\Lambda \rightarrow \infty} \frac{N(\Lambda)}{\Lambda} \leq 13<17 \leq \limsup _{\Lambda \rightarrow \infty} \frac{N(\Lambda)}{\Lambda}<20 .
$$

(d) For $\Lambda>0$ let $P_{\Lambda}$ denote the orthogonal projection onto the subspace of $H$ spanned by the eigenvectors of $D$ with non zero eigenvalues of numerical value at most $\Lambda$. For any any continuous function $f$ on $[0,1]$ we get

$$
\lim _{\Lambda \rightarrow \infty} \frac{1}{\log (\Lambda)} \operatorname{tr}\left(|D|^{-1} P_{\Lambda} \pi(f)\right)=\frac{10}{\log 2} \int_{0}^{1} f(x) d x .
$$

(e) For any ultrafilter $\omega$ on $\mathbf{N}$ and any continuous function $f$ on $[0,1]$ the Dixmier trace for this spectral triple is given by

$$
\operatorname{tr}_{\omega}\left(\pi(f)|D|^{-1}\right)=\frac{10}{\log 2} \int_{0}^{1} f(x) d x .
$$

A different choice of $\delta, \theta$ and $\rho$ would give a similar result, except for the constants $\log 2,10,13,17,20$.

\section{Details of the example}

The whole construction is based on the sets $\mathscr{T}_{n}$, which for a given $n$ is a set of centers for a minimal covering of the interval $[0,1]$ by balls of radius $\theta \rho^{n-1}=2^{1-n}$. So $\mathscr{T}_{1}=\mathscr{T}_{2}=\left\{2^{-1}\right\}$, and for

$$
n>2, \quad \mathscr{T}_{n}=\left\{(2 j+1) 2^{1-n} \mid 0 \leq j \leq 2^{n-2}-1\right\} .
$$

In particular the number of points $\left|\mathscr{T}_{n}\right|=2^{n-2}$ for $n \geq 2$. We can then prove the statement (a) without too much trouble. Let $u<v$ be points in $[0,1]$ and let $\varepsilon>0$ be given. Let $d_{\delta}$ denote the metric induced by the spectral triple. We then know from the theorem, that for any pair of points $u, v$ from the unit interval we have $|u-v| \leq d_{\delta}(u, v)$. Let then $f$ be a continuous function such that $\|[D, \pi(f)]\| \leq 1$, and let $\varepsilon>0$ be given. Then by the continuity of $f$ we can find a natural number $n$ and points $x_{j}, x_{k}$ from $\mathscr{T}_{n}$ such that $u \leq x_{j}<x_{k} \leq v$, $\left|f(u)-f\left(x_{j}\right)\right|<\varepsilon / 2$ and $\left|f(v)-f\left(x_{k}\right)\right|<\varepsilon / 2$. By the choice of $f$ we have

$$
\left|f\left(x_{k}\right)-f\left(x_{j}\right)\right| \leq \sum_{m=j}^{k-1}\left|f\left(x_{m+1}\right)-f\left(x_{m}\right)\right| \leq \sum_{m=j}^{k-1}\left|x_{m+1}-x_{m}\right|=x_{k}-x_{j} .
$$


Hence

$$
|f(v)-f(u)| \leq\left|f\left(x_{k}\right)-f\left(x_{j}\right)\right|+\varepsilon \leq\left|x_{k}-x_{j}\right|+\varepsilon \leq|v-u|+\varepsilon,
$$

and claim (a) follows.

The next task is then for a given natural number $n$ and each point $x$ in the set $\mathscr{T}_{n}$ to study the one-dimensional representation, say $\pi_{x}$, given as $\mathrm{C}([0,1]) \ni$ $f \rightarrow f(x)$ as a summand in the representation $\pi$ of the spectral triple. Suppose that a pair $\{x, y\}$ belongs to the index set $\mathscr{J}$ then both $\pi_{x}$ and $\pi_{y}$ will be counted once more in $\pi$ so the multiplicity of $\pi_{x}$ in $\pi$ is exactly the number of pairs $\{u, v\} \in \mathscr{J}$ which contains $x$. To find that number we will first determine the numbers $k_{0}$ and $l$ used in the proof of Theorem 3.2. According to the relation (3) we get $k_{0}=0$ and by (4) we get $l=0$ too. We then have to look at the rules (8) which defines the pairs in $\mathscr{J}$, and one gets for an $n \geq 5$ and an $x_{j}=$ $(2 j+1) 2^{1-n}$ in $\mathscr{T}_{n}$ that we have to look for points $v_{i}=(2 i+1) 2^{2-n} \in \mathscr{T}_{n-1}$, $y_{k}=(2 k+1) 2^{1-n} \in \mathscr{T}_{n}$ and $z_{m}=(2 m+1) 2^{-n} \in \mathscr{T}_{n+1}$ such that the pairs $\left\{v_{i}, x_{j}\right\},\left\{x_{j}, y_{k}\right\},\left\{x_{j}, z_{m}\right\}$ in which $x_{j}$ appears, are determined by the following relations

$$
\begin{aligned}
& \left|v_{i}-x_{j}\right| \leq \frac{3}{2} \cdot 2^{2-n}=6 \cdot 2^{-n} \\
& \left|x_{j}-y_{k}\right| \leq 4 \cdot 2^{1-n}=8 \cdot 2^{-n} \\
& \left|x_{j}-z_{m}\right| \leq \frac{3}{2} \cdot 2^{1-n}=3 \cdot 2^{-n} .
\end{aligned}
$$

There is a pattern for the computation of this, but it only holds for an $x_{j}$ which is not very near 0 or 1 . A detailed investigation shows that the regular pattern is valid for all $x_{j}$ for which $j \in\left\{2, \ldots, 2^{n-2}-3\right\}$, so it is only 4 points from each of the sets $\mathscr{T}_{n}$ which we will forget in the computations to come. Further it is not difficult to realize that the properties of the module is not dependent on any finite number of summands. Any finite number of two-point modules may be omitted without disturbing the properties of the module with respect to the metric or any of the asymptotic properties which we want to prove now. The reason for this is that any sort of property is always better described for a higher $n$. We may, and will, then assume that the module only starts by the summation of all the modules which involves points from $\mathscr{T}_{n}$ for $n \geq 5$. This means that some points from $\mathscr{T}_{4}$ will be introduced, as they are part of some two-point modules with points from $\mathscr{T}_{5}$. Let now $x_{j}=(2 j+1) 2^{1-n}$ be a point from $\mathscr{T}_{n}$ for an $n \geq 5$ and a $j$ such that $2 \leq j \leq 2^{n-2}-3$. We then get the 
following table for the number of points.

$\left|\left\{v_{i} \in \mathscr{T}_{n-1}|| v_{i}-x_{j} \mid \leq \frac{3}{2} \cdot 2^{2-n}\right\}\right|=2$

and the distances between $x_{j}$ and $v_{i}$

$=\left(2 \cdot 2^{-n}, 6 \cdot 2^{-n}\right)$.

$\left|\left\{y_{k} \in \mathscr{T}_{n}|| y_{k}-x_{j} \mid \leq 4 \cdot 2^{1-n}\right\}\right|=4$

and the distances between $x_{j}$ and $y_{k}$ and repeated if necessary

$=\left(4 \cdot 2^{-n}, 4 \cdot 2^{-n}, 8 \cdot 2^{-n}, 8 \cdot 2^{-n}\right)$.

$\left|\left\{z_{m} \in \mathscr{T}_{n+1}|| z_{m}-x_{j} \mid \leq \frac{3}{2} \cdot 2^{1-n}\right\}\right|=4$

and the distances between $x_{j}$ and $z_{m}$ and repeated if necessary $=\left(2^{-n}, 2^{-n}, 3 \cdot 2^{-n}, 3 \cdot 2^{-n}\right)$.

With respect to a point $x_{j}$ in $\mathscr{T}_{n}$ we then get that the multiplicity of $\pi_{x}$ is at most 10 , and it is 10 for all the points in $\mathscr{T}_{n}$ except for the 4 points nearest the boundary $\{0,1\}$.

We then turn to the absolute value $|D|$ of the Dirac operator. Let us then look at a pair $\{x, y\} \in \mathscr{J}$, then the Definition 2.1 shows that $\left|D_{x, y}\right|=|x-y|^{-1} I_{H_{x, y}}$ so a unit vector in the Hilbert space $H_{x, y}$ corresponding to the representation $\pi_{x}$ is an eigenvector for $|D|$ corresponding to the eigenvalue $|x-y|^{-1}$. This means that for an $x_{j}=(2 j+1) 2^{1-n}$, with $2 \leq j \leq 2^{n-2}-3$ the 10 eigenvalues for the operator $|D|$ corresponding to the summands of $\pi_{x_{j}}$ in $\pi$ will be; when listed by multiplicity and increasingly,

$$
\left\{2^{n-3}, 2^{n-3}, \frac{2^{n-1}}{3}, 2^{n-2}, 2^{n-2}, \frac{2^{n}}{3}, \frac{2^{n}}{3}, 2^{n-1}, 2^{n}, 2^{n}\right\} .
$$

We can now begin to compute the multiplicities of the eigenvalues for $|D|$. We know that all of the $2^{n-2}$ points $x_{j}$ in the set $\mathscr{T}_{n}$, except 4 , behave alike. For these 4 points the set of eigenvalues for $|D|$ are the same, but with lower multiplicities. In the computations to come it makes no difference, when estimating limits, to neglect this irregularity, so we will just assume that the 4 outmost points of each set $\mathscr{T}_{n}$ have the property that the absolute value of the Dirac operator follow the same eigenvalue pattern here, as for the regular points. From the list above we find that the only possible eigenvalues for $|D|$ on a vector corresponding to $\pi_{x_{j}}$ are of the forms $\frac{2^{k}}{3}$ and $2^{m}$. Further we see from the list, that the eigenvalue $2^{n}$ can only be associated with points from $\mathscr{T}_{n}, \mathscr{T}_{n+1}, \mathscr{T}_{n+2}$ and $\mathscr{T}_{n+3}$ and we get, since the number of points in a set $\left|\mathscr{T}_{k}\right|$ is $2^{k-2}$, that the multiplicity of the eigenvalue $2^{n}$ will be nearly

$$
2 \cdot 2^{n-2}+1 \cdot 2^{n-1}+2 \cdot 2^{n}+2 \cdot 2^{n+1}=7 \cdot 2^{n}
$$


Similarly the eigenvalue $\frac{2^{n}}{3}$, will be generated by points in $\mathscr{T}_{n}$ and $\mathscr{T}_{n+1}$ and the multiplicity of $\frac{2^{n}}{3}$ for $|D|$ is approximately

$$
2 \cdot 2^{n-2}+1 \cdot 2^{n-1}=2^{n} .
$$

We then get

$$
\operatorname{tr}\left(|D|^{-s}\right)=\sum_{n=5}^{\infty}\left(7 \cdot 2^{(n-n s)}+3^{s} \cdot 2^{(n-n s)}+E(n, s) 2^{-n s}\right)
$$

such that

$$
|E(n, s)| \leq 40 \cdot 3^{s} .
$$

It then follows that the module is summable for $s>1$ and not summable for $s=1$. The item (b) of the example is then proved, and we will begin to estimate $N(\Lambda)$, the number of eigenvalues for $|D|$, dominated by $\Lambda$. We can base the computation on the counting of the multiplicities which we just performed above. Let $\left\lfloor\frac{\log \Lambda}{\log 2}\right\rfloor$ denote the largest integer smaller than or equal to $\frac{\log \Lambda}{\log 2}$, then the number of eigenvalues $N(\Lambda)$ for $|D|$, which are dominated by $\Lambda$ is nearly

$$
\sum_{n=5}^{\left\lfloor\frac{\log \Lambda}{\log 2}\right\rfloor} 7 \cdot 2^{n}+\sum_{n=5}^{\left\lfloor\frac{\log 3 \Lambda}{\log 2}\right\rfloor} 2^{n}
$$

and we get

$$
N(\Lambda)=7 \cdot 2^{\left\lfloor\frac{\log \Lambda}{\log 2}\right\rfloor+1}+2^{\left\lfloor\frac{\log 3 \Lambda}{\log 2}\right\rfloor+1}+R(\Lambda)
$$

such that

$$
|R(\Lambda)|<40 \frac{\log \Lambda+3}{\log 2}+100<80 \log (\Lambda)+500 .
$$

We then get

$$
N(\Lambda)=\Lambda\left(7 \cdot 2^{\left(1-\left(\frac{\log \Lambda}{\log 2}-\left\lfloor\frac{\log \Lambda}{\log 2}\right\rfloor\right)\right)}+3 \cdot 2^{\left(1-\left(\frac{\log 3 \Lambda}{\log 2}-\left\lfloor\frac{\log 3 \Lambda}{\log 2}\right\rfloor\right)\right)}\right)+R(\Lambda) .
$$

The exponents for 2 in the expression above are always between 0 and 1 and they will vary discontinuously between these values so $\frac{N(\Lambda)}{\Lambda}$ has no limit for $\Lambda \rightarrow \infty$ and we get

$$
10 \leq \liminf _{\Lambda \rightarrow \infty} \frac{N(\Lambda)}{\Lambda} \leq 13<17 \leq \limsup _{\Lambda \rightarrow \infty} \frac{N(\Lambda)}{\Lambda} \leq 20
$$


This establishes the item (c) of the example. It seems likely that one can do better, but $\frac{\log 3}{\log 2}$ is not an integer so it is not possible to get down to 10 or up to 20.

Let us then turn to item (d). Let $\varepsilon>0$ and $f$ a continuous complex function on the interval $[0,1]$ be given. Since $f$ is continuous there exists a natural number $N_{1}$ such that

$$
\forall n \in \mathrm{N}, n \geq N_{1}:\left|\int_{0}^{1} f(x) d x-\sum_{j=0}^{2^{n-2}-1} f\left((2 j+1) 2^{1-n}\right) 2^{2-n}\right| \leq \frac{\varepsilon}{120}
$$

and

$$
40\|f\| 2^{2-n} \leq \frac{\varepsilon}{12}
$$

Then we define for any natural number $n \geq 5$ and any $j \in\left\{0, \ldots, 2^{n-2}-1\right\}$, the point $x_{j} \in \mathscr{T}_{n}$ as before by $x_{j}=(2 j+1) 2^{1-n}$ and the inequality (21) gives

$$
\forall n \in \mathrm{N}, n \geq N_{1}:\left|\int_{0}^{1} f(x) d x-\sum_{j=0}^{2^{n-2}-1} f\left(x_{j}\right) 2^{2-n}\right| \leq \frac{\varepsilon}{120} .
$$

Let now $Q_{n, j}$ be the orthogonal projection onto the span of the vectors in $H$ which have the property that each of them generates the one-dimensional representation $\pi_{x_{j}}$ of $\mathrm{C}([0,1])$. Let us then go back to the computations of the pairs which involved an $x_{j}$, and we find - again except for the 4 points which are closest to either 0 or $1-$ that for an $x_{j}$ from $\mathscr{T}_{n}$, the multiplicity of $\pi_{x_{j}}$ in $\pi$ is 10 and the corresponding 10 eigenvalues for $|D|^{-1}$ are

$$
\left\{2^{-n}, 2^{-n}, 2^{1-n}, 3 \cdot 2^{-n}, 3 \cdot 2^{-n}, 2^{2-n}, 2^{2-n}, 3 \cdot 2^{1-n}, 2^{3-n}, 2^{3-n}\right\}
$$

We can then for $2 \leq j \leq 2^{2 n-1}-3$ compute

$$
\operatorname{tr}\left(Q_{n, j}|D|^{-1}\right)=2^{-n}(1+1+2+3+3+4+4+6+8+8)=10 \cdot 2^{2-n},
$$

and for the 4 outmost points we get

$$
2^{2-n}<\operatorname{tr}\left(Q_{n, j}|D|^{-1}\right)<10 \cdot 2^{2-n},
$$

It then follows that for the projection $Q_{n}:=\sum_{j=0}^{2^{n-2}-1} Q_{n, j}$ and any continuous complex function $g$ on $[0,1]$ we have

$$
\operatorname{tr}\left(Q_{n} \pi(g)|D|^{-1}\right)=10 \sum_{j=0}^{2^{n-2}-1} g\left(x_{j}\right) 2^{2-n}+I(n)
$$


such that

$$
|I(n)| \leq 40\|g\| 2^{2-n}
$$

and we also get

$$
\left|\operatorname{tr}\left(Q_{n} \pi(g)|D|^{-1}\right)\right| \leq 10\|g\| .
$$

Hence for the given $f$ and a natural number $n \geq N_{1}$ we may use (22), (23), (24), (25) to obtain

$$
\left|\operatorname{tr}\left(Q_{n} \pi(f)|D|^{-1}\right)-10 \int_{0}^{1} f(x) d x\right| \leq \frac{\varepsilon}{6} .
$$

We can now introduce the positive real $\Lambda$ and we will choose it such that

$$
\Lambda \geq \exp \left(\frac{90\left(N_{1}+2\right)(\|f\|+1)}{\varepsilon}\right) \quad \text { and } \quad \Lambda \geq 2^{5}=32
$$

so

$$
\frac{\varepsilon}{90} \geq \frac{\left(N_{1}+2\right)(\|f\|+1)}{\log \Lambda} .
$$

Then we get for the given continuous function $f$ and for $M$ a natural number defined by $M:=\left\lfloor\frac{\log \Lambda}{\log 2}\right\rfloor$ that

$$
\frac{1}{\log \Lambda} \operatorname{tr}\left(P_{\Lambda} \pi(f)|D|^{-1}\right)=\frac{1}{\log \Lambda} \sum_{n=5}^{M} \operatorname{tr}\left(Q_{n} \pi(f)|D|^{-1}\right)+J(\Lambda)
$$

such that

$$
\begin{aligned}
|J(\Lambda)| & =\left|\frac{1}{\log \Lambda} \operatorname{tr}\left(\left(P_{\Lambda}-\sum_{n=5}^{M} Q_{n}\right) \pi(f)|D|^{-1}\right)\right| \\
& \leq\left|\frac{\|f\|}{\log \Lambda} \operatorname{tr}\left(\left(P_{\Lambda}-\sum_{n=5}^{M} Q_{n}\right)|D|^{-1}\right)\right|, \quad \text { by }(20) \\
& \leq\left|\frac{\|f\|}{\log \Lambda} \operatorname{tr}\left(\left(Q_{4}+Q_{M+1}+Q_{M+2}\right)|D|^{-1}\right)\right| \\
& \leq \frac{30\|f\|}{\log \Lambda} \\
& <\frac{\varepsilon}{90} .
\end{aligned}
$$


We can then continue as

$$
\begin{aligned}
\frac{1}{\log \Lambda} \sum_{n=5}^{M} \operatorname{tr}\left(Q_{n} \pi(f)|D|^{-1}\right) \\
=\frac{1}{\log \Lambda} \sum_{n=5}^{N_{1}} \operatorname{tr}\left(Q_{n} \pi(f)|D|^{-1}\right) \\
\quad+\frac{1}{\log \Lambda} \sum_{n=N_{1}+1}^{M} \operatorname{tr}\left(Q_{n} \pi(f)|D|^{-1}\right) \\
=\frac{M-N_{1}-1}{\log \Lambda} 10 \int_{0}^{1} f(x) d x+K(\Lambda)
\end{aligned}
$$

such that by (26), (27)

$$
|K(\Lambda)| \leq \frac{10\|f\| N_{1}}{\log \Lambda}+\frac{M-N_{1}-1}{\log \Lambda} \frac{\varepsilon}{6} \leq \frac{\varepsilon}{9}+\frac{\varepsilon}{6}<\frac{\varepsilon}{3} .
$$

The final estimation then becomes

$$
\begin{aligned}
& \left|\frac{1}{\log \Lambda} \operatorname{tr}\left(P_{\Lambda} \pi(f)|D|^{-1}\right)-\frac{10}{\log 2} \int_{0}^{1} f(x) d x\right| \text { by (31)-(35) } \\
& \leq\left|\frac{1}{\log \Lambda} \sum_{n=5}^{M} \operatorname{tr}\left(Q_{n} \pi(f)|D|^{-1}\right)-\frac{10}{\log 2} \int_{0}^{1} f(x) d x\right|+\frac{\varepsilon}{90} \text { by (36)-(39) } \\
& \leq\left|\frac{10\left(M-N_{1}-1\right)}{\log \Lambda}-\frac{10}{\log 2}\right|\left|\int_{0}^{1} f(x) d x\right|+\frac{\varepsilon}{3}+\frac{\varepsilon}{90} \\
& <10\|f\| \frac{\log \Lambda-\log 2\left(\left\lfloor\frac{\log \Lambda}{\log 2}\right\rfloor-N_{1}-1\right)}{\log 2 \log \Lambda}+\frac{\varepsilon}{2} \\
& \leq 10\|f\| \frac{\log \Lambda-\log 2\left(\frac{\log \Lambda}{\log 2}-1-N_{1}-1\right)}{\log 2 \log \Lambda}+\frac{\varepsilon}{2} \\
& =10\|f\| \frac{N_{1}+2}{\log \Lambda}+\frac{\varepsilon}{2} \operatorname{which} \text { by (29) } \\
& \leq \frac{\varepsilon}{9}+\frac{\varepsilon}{2}<\varepsilon .
\end{aligned}
$$

This establishes item (d) of the example and we will turn to look at item (e), but when we have a convergence as described in item (d), then it follows from 
the construction of the Dixmier trace [4] Chapter IV 2. $\beta$, pp. 303-308, that the limit obtained in (d) is the Dixmier trace of $\pi(f)|D|^{-1}$.

\section{REFERENCES}

1. Antonescu, C., Christensen, E., Metrics on group $C^{*}$-algebras and a non-commutative ArzelàAscoli theorem, J. Funct. Anal. 214 (2004), 247-259.

2. Christensen, E., Ivan, C., Spectral triples for $A F C^{*}$-algebras and metrics on the Cantor set, J. Operator Theory 56 (2006), 17-46.

3. Connes, A., Compact metric spaces, Fredholm modules, and hyperfiniteness, Ergodic Theory Dynam. Systems 9 (1989), 207-220.

4. Connes, A., Non-commutative Geometry, Academic Press, San Diego, 1994.

5. Connes, A., Unpublished notes on a Dirac operator associated to the Cantor subset of the unit interval.

6. Falconer, K. J., Fractal geometry, Mathematical Foundations and Applications, Wiley 1990.

7. Guido, D., Isola, T., Fractals in noncommutative geometry, in the Proceedings of the Conference, Mathematical Physics in Mathematics and Physics, Siena 2000, ed. Roberto Longo, Fields Inst. Commun. 30 (2001).

8. Guido, D., Isola, T., Dimensions and singular traces for spectral triples, with applications to fractals, J. Funct. Anal. 203 (2003), 362-400

9. Guido, D., Isola, T., Dimensions and spectral triples for fractals in $\mathrm{R}^{n}$, Advances in operator algebras and mathematical physics, Eds. F. Boca, O. Bratteli, R. Longo, H. Siedentop.

10. Kigami, J., Lapidus, M. L., Weyl's problem for the spectral distribution of Laplacians on p. c. f. self-similar fractals, Commun. Math. Phys. 158 (1993), 93-125.

11. Kadison, R. V., Ringrose, J. R., Fundamentals of the Theory of Operator Algebras, Academic Press, New York, 1983.

12. Lapidus, M. L., Fractal drum, inverse spectral problems for elliptic operators and a partial resolution of the Weyl-Berry conjecture, Trans. Amer. Math. Soc. 325 (1991), 465-529.

13. Lapidus, M. L., Vibrations of fractal drums, the Riemann hypothesis, waves in fractal media and the Weyl-Berry conjecture in Ordinary and Partial Differential Equations, ed. B. D. Sleeman and R. J. Jarvis, Pitmann Res. Notes Math. Ser. (289) 1993, 126-209.

14. Lapidus, M. L., Analysis on fractals, Laplacians on self-similar sets, noncommutative geometry and spectral dimensions, Topol. Methods Nonlinear Anal. 4 (1994), 137-195.

15. Lapidus, M. L., Towards a noncommutative fractal geometry? Laplacians and volume measures on fractals, in Harmonic Analysis and Nonlinear Differential Equations, Contemp. Math. 208 (1997), 211-252.

16. Lapidus, M. L., v. Frankenhuysen, M., Fractal Geometry and Number Theory, Birkhäuser, Boston, 2000.

17. Lapidus, M. L., Maier, H., The Riemann hypothesis and inverse spectral problems for fractal strings, J. London Math. Soc. 52 (1995), 15-34.

18. Lapidus, M. L., Pomerance, C., The Riemann zeta-function and the one-dimensional WeylBerry conjecture for fractal drums, Proc. London Math. Soc. 66 (1993), 41-69.

19. Ozawa, N., Rieffel, M. A., Hyperbolic group $C^{*}$-algebras and free product $C^{*}$-algebras as compact quantum metric spaces, Canad. J. Math. 57 (2005), 1056-1079.

20. Pavlović, B., Defining metric spaces via operators from unital $C^{*}$-algebras, Pacific J. Math. 186 (1998), 285-313.

21. Rieffel, M. A., Comments concerning non-commutative metrics, Talk given at AMS Special Session, Texas A\&M, October 1993.

22. Rieffel, M. A., Metrics on states from actions of compact groups, Doc. Math. 3 (1998), 215-229. 
23. Rieffel, M. A., Metrics on state spaces, Doc. Math. 4 (1999), 559-600.

24. Rieffel, M. A., Compact quantum metric spaces, Operator Algebras, quantization, and noncommutative geometry, Contemp. Math. 365 (2004), 315-330.

25. Schrohe, Elmar, Walze, Markus, and Warzecha, Jan-Martin, Construction de triplets spectraux à partir de modules de Fredholm, C. R. Acad. Sci. Paris Sér. I Math. 326 (1998), no. 10, $1195-1199$.

DEPARTMENT OF MATHEMATICS UNIVERSITY OF COPENHAGEN DENMARK

E-mail: echris@math.ku.dk
DEPARTMENT OF MATHEMATICS

UNIVERSITY OF HANNOVER

GERMANY

E-mail: antonescu@math.uni-hannover.de 